\title{
Motion of a classical charged particle
}

\author{
J. M. Aguirregabiria, ${ }^{1}{ }$. Llosa, ${ }^{2, *}$ and A. Molina ${ }^{2}$ \\ ${ }^{1}$ Fisika Teorikoa, Zientzia eta Teknologia Fakultatea, Euskal Herriko Unibertsitatea, Spain \\ ${ }^{2}$ Departament de Física Fonamental, Universitat de Barcelona ${ }^{\dagger}$, Spain
}

(Received 23 January 2006; published 19 June 2006)

\begin{abstract}
The Lorentz-Dirac equation is not an unavoidable consequence of solely linear and angular momenta conservation for a point charge. It also requires an additional assumption concerning the elementary character of the charge. We here use a less restrictive elementarity assumption for a spinless charge and derive a system of conservation equations that are not properly the equation of motion because, as it contains an extra scalar variable, the future evolution of the charge is not determined. We show that a supplementary constitutive relation can be added so that the motion is determined and free from the troubles that are customary in the Lorentz-Dirac equation, i.e., preacceleration and runaways.
\end{abstract}

DOI: 10.1103/PhysRevD.73.125015

\section{INTRODUCTION}

The Lorentz-Dirac equation is widely accepted as the classical equation for motion of an elementary point charge interacting with its own radiation (see for instance [1-4]):

$$
m a^{\mu}=F^{\mu}+\frac{2 e^{2}}{3 c^{3}}\left(\dot{a}^{\mu}-\frac{1}{c^{2}} a^{\lambda} a_{\lambda} v^{\mu}\right)
$$

where $F^{\mu}=\frac{e}{c} F_{\mathrm{ext}}^{\mu \nu} v_{\nu}$ is the external electromagnetic force.

It is also well known that this equation is affected by some irreconcilable difficulties that already show up in the case of rectilinear motion. Consider a free point charge that enters perpendicularly a parallel-plate capacitor at $\tau=0$ (proper time) and leaves it at $\tau_{1}>0$. For $\tau<0$ the charge is free, $F^{\mu}=0$, and the solution to (1) is a uniform rectilinear motion, $a^{\mu}=0$. We can therefore take $a^{\mu}(0)=0$ and $v^{\mu}(0)=v_{\text {in }}^{\mu}$ as initial data to integrate Eq. (1), so obtaining a unique solution for the velocity $v^{\mu}$. Nevertheless, this solution has the drawback that, not only does $a^{\mu}(\tau)$ not vanish for $\tau>\tau_{1}$ (when the external action has ceased), but it grows exponentially for $\tau \rightarrow \infty$, what is known as a runaway solution.

Rohrlich [2] put forward a way out consisting in that (1) is not the equation of motion, but it must be supplemented with an asymptotic condition: if the external force $F^{\mu}$ asymptotically vanishes, then the acceleration $a^{\mu}$ asymptotically vanishes too. The resulting equation of motion is of integro-differential type and runaway solutions are ruled out (see also [5]).

This alternative however implies what is called preacceleration. Although the external force vanishes for $\tau<0$, the solution to the above integro-differential equation presents nonvanishing acceleration before the force starts. This is not a surprising feature because, as is pointed out in [6], it is a consequence of demanding the asymptotic condition in the future: the integro-differential equation

\footnotetext{
*Electronic address: pitu.llosa@ub.edu

†Postal address: Martí i Franquès, 1; E-08028 Barcelona, Spain.
}

PACS numbers: 03.50.De, 05.45. $-\mathrm{a}, 41.60 .-\mathrm{m}, 45.30 .+\mathrm{s}$

of motion itself "foresees" what will happen in the future, $\tau>\tau_{1}$.

It thus seems as though we were facing the following dilemma [6]: either (a) classical electrodynamics is selfcontradictory or (b) the Lorentz-Dirac equation is not the right equation that follows from classical electrodynamics.

In view of this dilemma different stances are found in the literature. Rohrlich [2] adopts the alternative (a) and adds that this is not a major trouble because the time scale at which preacceleration shows up is too small $\left(\tau_{0} \approx 10^{-23} \mathrm{~s}\right.$ for electrons) far beyond the limits of validity of the classical theory. He further stresses that [7] "the notion of 'classical point charge' is an oxymoron ..." because classical physics ceases to be valid below Compton wavelength. Moniz and Sharp also argued [8-10] that classical electrodynamics is only consistent in describing the motion of charges with radius larger than the classical electron radius, while the quantum theory of nonrelativistic charges is free of runaways and preacceleration.

Other authors [11,12] embrace the alternative (b) on the basis that the derivation of the Lorentz-Dirac equation involves Taylor expansions and therefore presumes that both the charge world line and the external force are analytic functions. As a consequence, Eq. (1) is not valid in those points where $x^{\mu}(\tau)$ and $f^{\mu}(\tau)$ are not analytic. Particularly, Yaghjian [12] studies a charged spherical shell of radius $\epsilon$ and obtains an alternative equation:

$$
m a^{\mu}=f^{\mu}+\frac{2 e^{2}}{3 c^{3}} \eta(\tau)\left(\dot{a}^{\mu}-\frac{1}{c^{2}} a^{\lambda} a_{\lambda} v^{\mu}\right)
$$

where $\eta(\tau)=0$ for $\tau<0$ and $\eta(\tau)=1$ for $\tau \geq 2 \epsilon / c$. In another approach [13-19], the Lorentz-Dirac equation is taken as a necessary - but not sufficient - condition the true equation of motion must fulfill. The true equation of motion, which will not have neither preacceleration nor runaway solutions, is of second order and can only be constructed by using a series expansion or a method of successive approximations. 
Some authors [20] consider that the behavior of the solution of the Lorentz-Dirac equation is not unphysical but a consequence of the negative mechanical mass necessary to renormalize the infinite electromagnetic mass. Others [21] consider that the commented difficulties are not real physical problems, as they accept that acceleration can have a singularity in points where the applied force has a discontinuity.

None of these justifications is fully satisfactory to us. Indeed, consider a classical charge modeled by a charge distribution and the corresponding matter distribution inside a sphere of radius $\epsilon$. Provided that a suitable set of constitutive relations and state equations is added, the local conservation of energy momentum yields an evolution law for this continuous medium, which is deterministic and causal: the electric current and the matter variables distribution at $t=0$ determine the future values of these magnitudes. It is, to say the least, surprising that, somewhere in the way to the limit $\epsilon \rightarrow 0$, the causal and deterministic nature of the classical problem is lost.

The Lorentz-Dirac equation is often presented as an unavoidable and flawless consequence of classical electrodynamics plus the local conservation of total energy and momentum $[1,2,4]$. However, as the electromagnetic field contribution $\Theta^{\mu \nu}$ to the energy-momentum tensor is singular on the charge's world line-it behaves as $\mathrm{O}\left(r^{-4}\right)$ some creative "tricks" are needed to appropriately handle such a singular behavior in the energy-momentum balance. In our opinion, in most approaches to this problem some additional assumption slips into the reasoning through one of these tricks.

In this context, it is worth mentioning Rowe's work $[3,22]$, where more elaborated mathematical tools, namely, regularization of generalized functions, are used to properly handle the singularity in $\Theta^{\mu \nu}$ and obtain the LorentzDirac equation. The use of generalized functions (or distributions) has also the advantage that no mass renormalization is necessary.

Using similar mathematical tools Lozada [23] derives a general class of equations on the sole assumption of linear and angular momenta conservation. Only the requirement is added that the multipole structure of the particle is as simple as possible. These equations are actually constraints connecting the kinematical variables of the charge with its mass and some other quantities (of multipole character). Then, if some phenomenological relations relating these multipole quantities with kinematical variables are introduced by hand, the different equations of motion present in the literature $[1,24,25]$ are obtained.

We shall here use these same mathematical tools to review the derivation of the Lorentz-Dirac equation and illustrate that it is not a straight consequence of classical electrodynamics plus energy-momentum conservation, but it rather relies on the special way that the elementary character of the charge is implemented.
We shall here describe a point charge as a current distribution in an extended material body in the limit when the radius $\epsilon \rightarrow 0$. The total energy-momentum tensor results from two contributions: an electromagnetic part $\Theta^{\mu \nu}$, which is associated to the field and pervades spacetime, and a material part $K^{\mu \nu}$, which we assume confined to a world tube of radius $\epsilon$ and accounts for kinetic energy and the stresses that balance the electric repulsion among the parts of a neat total charge confined in a small volume.

For $\epsilon>0$ both contributions $\Theta^{\mu \nu}$ and $K^{\mu \nu}$ are continuous functions and can be considered separately. But in the limit $\epsilon \rightarrow 0$, the electromagnetic part presents a singularity $\mathrm{O}\left(r^{-4}\right)$ on the world line. Therefore, in the limit $\epsilon \rightarrow 0$ none of these two contributions can be properly defined, even resorting to generalized functions. However, nothing forbids the total energy-momentum tensor to converge to a generalized function for $\epsilon \rightarrow 0$, which will likely include $\delta$ functions and its derivatives on the point charge world line.

Moreover, our approach does not need assuming that the involved functions are analytic. Although Taylor expansions to some finite order are used, these hold for functions that are smooth enough, without need of analyticity [26].

We shall examine what restrictions on the charge's motion follow from local conservation of linear and angular momenta and find that the result is not the LorentzDirac equation but a wide class of equations of motion [23] that, in addition to kinematical variables, involve a few dipolelike variables (even for a point charge). In Sec. IVA the condition that the point charge does not have spin is then obtained by requiring that the Pauli-Lubanski vector vanishes. This reduces the range of the class of equations of motion but, contrary to what other authors obtain [23], spin zero does not inevitably lead to the Lorentz-Dirac equation.

Our conclusion is that linear and angular momenta conservation plus the additional "elementarity condition" are not restrictive enough to determine a unique equation of motion for a spinless charged particle. We shall see that to achieve a classical law of motion a supplementary (phenomenological) constitutive relation is needed.

We shall finally illustrate, by an example of onedimensional motion, that constitutive relations exist such that the resulting law of motion admits solutions that are free of both preacceleration or runaways.

\section{STATEMENT OF THE PROBLEM}

\section{A. Notation}

The retarded Liénard-Wiechert field of a point charge has an outstanding role along the present paper. Therefore it will be helpful to use retarded optical coordinates [27] (as in Ref. [3]) based on a timelike world line $\Gamma \equiv\left\{z^{\mu}(\tau)\right\}$ and an orthonormal tetrad $\left\{e_{(\alpha)}^{\mu}\right\}_{\alpha=1,2,3,4}$, which is FermiWalker transported along $\Gamma$, 


$$
\frac{d e_{(\alpha)}^{\mu}}{d \tau}=\left[v^{\mu} a_{\nu}-v_{\nu} a^{\mu}\right] e_{(\alpha)}^{\nu} .
$$

With a properly chosen initial tetrad, the latter evolution equation is consistent with the conditions

$$
\begin{aligned}
& e_{(\alpha)}^{\mu} e_{(\beta)}^{\nu} \eta_{\mu \nu}=\eta_{\alpha \beta}, \\
& e_{(4)}^{\mu}=v^{\mu}=\dot{z}^{\mu}, \quad \text { and } \quad a^{\mu}=\dot{v}^{\mu},
\end{aligned}
$$

where a dot means "derivative with respect to $\tau$ " and $\eta_{\mu \nu}=(+++-)$. Moreover, from now on we use units such that $c=1$.

For any point $x$ in spacetime, the equation

$$
\left[x^{\mu}-z^{\mu}(\tau)\right]\left[x^{\nu}-z^{\nu}(\tau)\right] \eta_{\mu \nu}=0,
$$

supplemented with $x^{4}>z^{4}(\tau)$, always has a unique solution, $\tau=\tau(x)$, which defines a time coordinate for $x$.

The space coordinates are

$$
X^{i}=e_{(i)}^{\mu}\left(x_{\mu}-z_{\mu}[\tau(x)]\right)
$$

and the inverse coordinate transformation then reads

$$
x^{\mu}=z^{\mu}(\tau)+\rho v^{\mu}(\tau)+X^{i} e_{(i)}^{\mu}(\tau),
$$

where $\rho=\|\vec{X}\|=\sqrt{\left(X^{1}\right)^{2}+\left(X^{2}\right)^{2}+\left(X^{3}\right)^{2}}$.

The following relations and quantities, introduced in Ref. [4], will be useful hereafter:

$$
\left.\begin{array}{c}
\rho=-\left[x_{\mu}-z_{\mu}(\tau)\right] v^{\mu}(\tau), \quad k^{\mu}:=\frac{1}{\rho}\left[x^{\mu}-z^{\mu}(\tau)\right], \\
n^{\mu}:=k^{\mu}-v^{\mu}, \quad n^{\mu} n_{\mu}=1, \quad k_{\mu} v^{\mu}=-1,
\end{array}\right\}
$$

$$
\partial_{\mu} \rho=n_{\mu}+\rho\left(a^{\alpha} n_{\alpha}\right) k_{\mu} .
$$

The unit space vector $n^{\mu}$ can be written as

$$
n^{\mu}=\frac{X^{i}}{\rho} e_{(i)}^{\mu} \equiv \hat{n}^{i} e_{(i)}^{\mu}
$$

Finally, the volume elements in Lorentzian and in retarded optical coordinates are related by

$$
d^{4} x=d \tau d^{3} \vec{X}=\rho^{2} d \tau d \rho d^{2} \Omega(\hat{n}),
$$

where $d^{2} \Omega(\hat{n})$ is the solid angle element.

\section{B. Some definitions and postulates}

A point charge is described by a current density fourvector $j^{\mu}$, and an energy-momentum tensor $t^{\mu \nu}$, fulfilling

$$
\partial_{\mu} j^{\mu}=0, \quad \partial_{\mu} t^{\mu \nu}=0, \quad \text { and } \quad t^{\mu \nu}=t^{\nu \mu},
$$

respectively, the local conservation laws for total electric charge, energy momentum, and angular momentum.

In our model $j^{\mu}$ and $t^{\mu \nu}$ are expected to be the limit of a continuous distribution of charge and energy momentum when the radius goes to zero, namely,
(1) an electric current vector $J^{\mu}(\epsilon ; x)$, which is confined to an "optical tube" of radius $\epsilon$ around a timelike world line $\Gamma$, that is,

$$
\rho(x)>\epsilon \Rightarrow J^{\mu}(\epsilon ; x)=0,
$$

where $\rho(x)$ is given by (7),

(2) an energy-momentum tensor $T^{\mu \nu}(\epsilon ; x)$ which results from two contributions:

$$
T^{\mu \nu}(\epsilon ; x)=\Theta^{\mu \nu}(\epsilon ; x)+K^{\mu \nu}(\epsilon ; x) .
$$

The first term comes from the total electromagnetic field:

$$
F^{\mu \nu}(\epsilon ; x)=F_{R}^{\mu \nu}(\epsilon ; x)+F_{\mathrm{ext}}^{\mu \nu}(x),
$$

namely, the sum of the retarded solution of the Maxwell equations for the current $J^{\mu}(\epsilon ; x)$ plus an external free electromagnetic field. The second term in (12) comes from the matter distribution which is also confined to the above mentioned "optical tube":

$$
\rho(x)>\epsilon \Rightarrow K^{\mu \nu}(\epsilon ; x)=0 .
$$

The above continuous distributions of electric current and energy momentum are assumed to fulfill the local conservation laws:

$$
\partial_{\mu} J^{\mu}=0, \quad \partial_{\mu} T^{\mu \nu}=0, \quad T^{\mu \nu}=T^{\nu \mu} .
$$

We shall assume that both $J^{\mu}(\epsilon ; x)$ and $K^{\mu \nu}(\epsilon ; x)$ are locally summable in $\mathbb{R}^{4}$ and that $F_{\mathrm{ext}}^{\mu \nu}(x)$ is continuous in $\mathbb{R}^{4}$.

The retarded electromagnetic field is given by [2]

$$
\begin{aligned}
F_{R}^{\mu \nu}(\epsilon ; x) & =8 \pi \int J^{[\nu}\left(\epsilon ; x^{\prime}\right) \partial^{\mu]} D_{R}\left(x-x^{\prime}\right) d^{4} x^{\prime} \quad \text { with } \\
D_{R}(x) & =\frac{1}{2 \pi} Y\left(x^{4}\right) \delta\left(x^{\rho} x_{\rho}\right) .
\end{aligned}
$$

$\left(Y\left(x^{4}\right)\right.$ is the Heaviside step function.) It is thus a continuous function and therefore locally summable in $\mathbb{R}^{4}$.

In turn, the electromagnetic contribution to the energymomentum tensor,

$$
\begin{aligned}
\Theta^{\mu \nu}(\epsilon ; x)= & \frac{1}{4 \pi}\left[F^{\mu \alpha}(\epsilon ; x) F_{\alpha}^{\nu}(\epsilon ; x)\right. \\
& \left.-\frac{1}{4} \eta^{\mu \nu} F^{\rho \alpha}(\epsilon ; x) F_{\rho \alpha}(\epsilon ; x)\right],
\end{aligned}
$$

is also locally summable.

The mathematical framework where the limits for $\epsilon \rightarrow 0$ of $J^{\mu}(\epsilon ; x)$ and $T^{\mu \nu}(\epsilon ; x)$ are meaningful and can be appropriately handled is the space $\mathcal{D}^{\prime}\left(\mathbb{R}^{4}\right)$ of generalized functions [28,29]. As locally summable functions, $J^{\mu}(\epsilon ; x)$ and $T^{\mu \nu}(\epsilon ; x)$ can be associated to generalized functions and, provided that the limits 


$$
j^{\mu}=\lim _{\epsilon \rightarrow 0} J^{\mu}(\epsilon), \quad t^{\mu \nu}=\lim _{\epsilon \rightarrow 0} T^{\mu \nu}(\epsilon)
$$

exist in $\mathcal{D}^{\prime}\left(\mathbb{R}^{4}\right)$, the continuity of differentiation operators in $\mathcal{D}^{\prime}\left(\mathbb{R}^{4}\right)$ [29] guarantees the conservation laws (10) as the limit of (15) for $\epsilon \rightarrow 0$.

These conservation laws must now be understood in the sense of $\mathcal{D}^{\prime}\left(\mathbb{R}^{4}\right)$, i.e., $\left(\partial_{\mu} j^{\mu}, \varphi\right)=0$ and $\left(\partial_{\mu} t^{\mu \nu}, \varphi\right)=0$, for any $\varphi \in \mathcal{D}\left(\mathbb{R}^{4}\right)$, or

$$
\left(j^{\mu}, \partial_{\mu} \varphi\right)=0 \quad \text { and } \quad\left(t^{\mu \nu}, \partial_{\mu} \varphi\right)=0 .
$$

\section{THE POINT CHARGE LIMIT}

\section{A. The electric current}

If the support of $J^{\mu}(\epsilon ; x)$ is the "optical tube" $\rho(x) \leq \epsilon$, then for any $\varphi \in \mathcal{D}\left(\mathbb{R}^{4}\right)$ such that $\operatorname{supp} \varphi$ does not intersect the world line $\Gamma$, it exists $\epsilon_{1}>0$ such that $\varphi(x)=0$ whenever $\rho(x) \leq \epsilon_{1}$. Therefore, for all $\epsilon<\epsilon_{1}$,

$$
\left(J^{\mu}(\epsilon), \varphi\right)=\int d^{4} x J^{\mu}(\epsilon ; x) \varphi(x)=0,
$$

and in the limit $\epsilon \rightarrow 0$ it follows that

$\left(j^{\mu}, \varphi\right)=0, \quad \forall \varphi \in \mathcal{D}\left(\mathbb{R}^{4}\right)$ such that $\Gamma \cap \operatorname{supp} \varphi=\varnothing$.

The support of the generalized function $j^{\mu}$ is therefore confined to the world line $\Gamma$ and, according to a wellknown result on generalized functions [29], $j^{\mu}$ can be written as a sum of $\delta$-functions and its derivatives up to a finite order:

$$
\begin{aligned}
j^{\mu}= & \int d \tau\left[l^{\mu}(\tau) \delta(x-z(\tau))+l^{\alpha \mu}(\tau) \partial_{\alpha} \delta(x-z(\tau))\right. \\
& \left.+\ldots+l^{\alpha_{1} \ldots \alpha_{n} \mu}(\tau) \partial_{\alpha_{1} \ldots \alpha_{n}} \delta(x-z(\tau))\right],
\end{aligned}
$$

with $l^{\left(\alpha_{1} \ldots \alpha_{r}\right) \mu} v_{\alpha_{1}}=0 ; r=1, \ldots n$.

To model a point charge we only keep the lowest order term and, as a consequence of the conservation law (10), we have [4]

$$
j^{\mu}=e \int d \tau v^{\mu}(\tau) \delta(x-z(\tau)),
$$

where $e$ is the electric charge of the particle and is a constant scalar.

\section{B. The energy-momentum tensor}

In our approach, the limits for $K^{\mu \nu}(\epsilon)$ and $\Theta^{\mu \nu}(\epsilon)$ do not need to exist separately in $\mathcal{D}^{\prime}\left(\mathbb{R}^{4}\right)$ and only the joint limit is assumed to be physically meaningful:

$$
t^{\mu \nu}=\lim _{\epsilon \rightarrow 0}\left[K^{\mu \nu}(\epsilon)+\Theta^{\mu \nu}(\epsilon)\right] \in \mathcal{D}^{\prime}\left(\mathbb{R}^{4}\right) .
$$

This fact expresses the notion that, although in the separate limits for both $K^{\mu \nu}(\epsilon)$ and $\Theta^{\mu \nu}(\epsilon)$ some infinities on the world line $\Gamma$ could arise, these cancel each other, so that $t^{\mu \nu}$ is well defined in $\mathcal{D}^{\prime}\left(\mathbb{R}^{4}\right)$.

\section{The matter contribution}

If we restrict to test functions $\varphi \in \mathcal{D}\left(\mathbb{R}^{4}-\Gamma\right)$, we have that

$$
\lim _{\epsilon \rightarrow 0} K^{\mu \nu}(\epsilon)=0 \in \mathcal{D}^{\prime}\left(\mathbb{R}^{4}-\Gamma\right) .
$$

Indeed, for any $\varphi \in \mathcal{D}\left(\mathbb{R}^{4}-\Gamma\right)$ it exists $\epsilon_{1}>0$ such that $\varphi(x)=0$ whenever $\rho(x) \leq \epsilon_{1}$. The confinement condition (14) then implies that

$$
\forall \epsilon<\epsilon_{1}, \quad\left(K^{\mu \nu}(\epsilon), \varphi\right)=\int d^{4} x K^{\mu \nu}(\epsilon ; x) \varphi(x)=0
$$

and Eq. (22) follows [29].

\section{The electromagnetic contribution}

Recall now Eqs. (16) and (17). We have the pointwise limit $\forall x \notin \Gamma$

$$
\lim _{\epsilon \rightarrow 0} F^{\mu \nu}(\epsilon ; x)=F_{R}^{\mu \nu}(x)+F_{\mathrm{ext}}^{\mu \nu}(x),
$$

where $F_{R}^{\mu \nu}(x)$ is the retarded Liénard-Wiechert field. It can be written as the sum of the radiation field plus the velocity field:

$$
F_{R}^{\mu \nu}(x)=F_{\mathrm{I}}^{\mu \nu}(x)+F_{\mathrm{II}}^{\mu \nu}(x),
$$

where, in the notation introduced in Subsection II A (also in Ref. [4]):

$$
\begin{gathered}
F_{\mathrm{I}}^{\mu \nu}(x)=\frac{e}{\rho}\left[(a k) v^{\mu} \wedge k^{\nu}+a^{\mu} \wedge k^{\nu}\right], \\
F_{\mathrm{II}}^{\mu \nu}(x)=\frac{e}{\rho^{2}} v^{\mu} \wedge k^{\nu},
\end{gathered}
$$

where $\wedge$ means the skewsymmetric product: $v^{\mu} \wedge k^{\nu} \equiv$ $2 v^{[\mu} k^{\nu]}=v^{\mu} k^{\nu}-v^{\nu} k^{\mu}$ and $(a k) \equiv a^{\lambda} k_{\lambda}$.

Similarly, for the electromagnetic energy-momentum tensor we have the pointwise convergence:

$$
\lim _{\epsilon \rightarrow 0} \Theta^{\mu \nu}(\epsilon ; x)=\Theta^{\mu \nu}(x), \quad \forall x \notin \Gamma .
$$

As a consequence of (23), $\Theta^{\mu \nu}(x)$ can be split as

$$
\Theta^{\mu \nu}(x)=\Theta_{R}^{\mu \nu}(x)+\Theta_{\mathrm{ext}}^{\mu \nu}(x)+\Theta_{\text {mix }}^{\mu \nu}(x) .
$$

The first and second terms in the right-hand side (r.h.s.) respectively result from substituting $F_{R}^{\mu \nu}(x)$ and $F_{\text {ext }}^{\mu \nu}(x)$ into the quadratic expression (17), whereas $\Theta_{\text {mix }}^{\mu \nu}(x)$ comes from the cross terms.

$\Theta_{\text {mix }}^{\mu \nu}(x)$ and $\Theta_{\text {ext }}^{\mu \nu}(x)$ are locally summable in $\mathbb{R}^{4}$. This is obvious for $\Theta_{\mathrm{ext}}^{\mu \nu}(x)$ because it is continuous everywhere. As for $\Theta_{\text {mix }}^{\mu \nu}(x)$, it is a sum of products of $F_{\mathrm{ext}}^{\mu \nu}(x)$, which is continuous, and $F_{R}^{\mu \nu}(x)$, which is also continuous except for a singularity of order $\rho^{-2}$ on $\Gamma$ that is cancelled by the factor $\rho^{2}$ in the volume element (9). Therefore, $\Theta_{\text {mix }}^{\mu \nu}(x)$ is also locally summable in $\mathbb{R}^{4}$. We shall, respectively, denote: 


$$
\theta_{\mathrm{ext}}^{\mu \nu}:=\lim _{\epsilon \rightarrow 0} \Theta_{\mathrm{ext}}^{\mu \nu}(\epsilon ; x) \quad \text { and } \quad \theta_{\mathrm{mix}}^{\mu \nu}:=\lim _{\epsilon \rightarrow 0} \Theta_{\mathrm{mix}}^{\mu \nu}(\epsilon ; x)
$$

with $\theta_{\text {ext }}, \theta_{\text {mix }} \in \mathcal{D}^{\prime}\left(\mathbb{R}^{4}\right)$.

On its turn, the $\Theta_{R}^{\mu \nu}(x)$ contribution can be written as [4]

$$
\begin{aligned}
\Theta_{R}^{\mu \nu}(x)= & \frac{e^{2}}{4 \pi \rho^{4}}\left[v^{\mu} k^{\nu}+v^{\nu} k^{\mu}+\frac{1}{2} \eta^{\mu \nu}-k^{\mu} k^{\nu}\right] \\
& +\frac{e^{2}}{4 \pi \rho^{3}}\left[a^{\mu} k^{\nu}+a^{\nu} k^{\mu}-(a n)\left(n^{\mu} k^{\nu}+n^{\nu} k^{\mu}\right)\right] \\
& +\frac{e^{2}}{4 \pi \rho^{2}}\left[a^{2}-(a n)^{2}\right] k^{\mu} k^{\nu},
\end{aligned}
$$

which is continuous for $x \notin \Gamma$ but, owing to the $\rho^{-4}$ and $\rho^{-3}$ singularities on the r.h.s., it not only has a singularity on $\Gamma$, but in addition it is not locally summable. Therefore, no generalized function in $\mathcal{D}^{\prime}\left(\mathbb{R}^{4}\right)$ can be associated to $\Theta_{R}^{\mu \nu}(x)$ in the standard way.

Now, since $\Theta_{R}^{\mu \nu}(x)$ is a continuous function on $\mathbb{R}^{4}-\Gamma$, it is locally summable there, and this allows to take its finite part $\theta_{R}^{\mu \nu} \in \mathcal{D}^{\prime}\left(\mathbb{R}^{4}\right)[28,29]$ :

$$
\begin{aligned}
\left(\theta_{R}^{\mu \nu}, \varphi\right):= & \int d^{4} x \Theta_{R}^{\mu \nu}(x)\{\varphi(x)-Y(L-\rho) \\
& \left.\times\left[\varphi(z)+\rho k^{\alpha} \partial_{\alpha} \varphi(z)\right]\right\}
\end{aligned}
$$

for any $\varphi \in \mathcal{D}^{\prime}\left(\mathbb{R}^{4}\right)$, where $L$ is an arbitrary chosen length scale, $z=z(\tau(x))$ and $\tau(x), k^{\alpha}$ and $\rho(x)$ are defined in (4) and (7).

Some points concerning the definition (30) are worth commenting:

(i) The integral in the r.h.s. converges. Indeed, on the one hand, for $\rho>L, \Theta_{R}^{\mu \nu}(x)$ is continuous and $\varphi(x)$ has compact support and, on the other, inside $\rho \leq L$ we can use the mean value Taylor theorem [26] for the smooth function $\varphi$ :

$$
\begin{aligned}
\varphi(x)= & \varphi(z)+\rho k^{\lambda} \partial_{\lambda} \varphi(z) \\
& +\frac{1}{2} \rho^{2} k^{\lambda} k^{\mu} \partial_{\lambda \mu} \varphi\left(z+\rho^{\prime} k\right),
\end{aligned}
$$

with $0<\rho^{\prime}<\rho(x)$. Now, since $\varphi$ is smooth and has compact support, $\partial_{\lambda \mu} \varphi$ is bounded and it exists $M>$ 0 such that

$$
\begin{aligned}
& \left|\varphi(x)-\left[\varphi(z)+\rho k^{\lambda} \partial_{\lambda} \varphi(z)\right]\right|<M \rho^{2}, \\
& x \in \operatorname{supp} \varphi, \quad 0 \leq \rho \leq L .
\end{aligned}
$$

Hence the integrand in the r.h.s. of (30) presents a singularity of order $\rho^{-2}$ on $\Gamma$ and therefore the integral converges.

(ii) For a test function $\varphi \in \mathcal{D}\left(\mathbb{R}^{4}-\Gamma\right)$, the function and all its derivatives vanish on $\Gamma$. Hence, (30) amounts to

$$
\left(\theta_{R}^{\mu \nu}, \varphi\right)=\int d^{4} x \Theta_{R}^{\mu \nu}(x) \varphi(x)<+\infty,
$$

that is, the restriction of $\theta_{R}^{\mu \nu}$ to test functions in $\mathcal{D}\left(\mathbb{R}^{4}-\Gamma\right)$ coincides with $\Theta_{R}^{\mu \nu}(x)$.

(iii) The definition (30) consists of eliminating from the integrand as many terms in the Taylor expansion of $\varphi(x)$ as necessary, in such a way that the remainder is summable and the condition (ii) above is fulfilled. As a consequence, the finite part $\theta_{R}^{\mu \nu} \in$ $\mathcal{D}^{\prime}\left(\mathbb{R}^{4}\right)$ is not unique. Indeed, on the one hand, we could have subtracted some more terms in the Taylor expansion of $\varphi$, and obtained a convergent integral also fulfilling the requirement (ii). Besides, the length scale $L$ is quite arbitrary and could even depend on $\tau(x)$.

This results in that $\theta_{R}^{\mu \nu}$ is determined up to a finite sum of $\Gamma$-supported $\delta$-functions and their derivatives, multiplied by arbitrary $\tau$-dependent coefficients, in an expression similar to (19). This lack of uniqueness in the definition of $\theta_{R}^{\mu \nu}$ is not relevant for us (as it is for Rowe [22]). Our derivation of a law of motion relies on the local conservation of the total energy-momentum tensor $t^{\mu \nu}$ and it does not depend on a particular identification of the matter and field contributions.

To give a more specific expression for $\theta_{R}^{\mu \nu}$, we realize that since the r.h.s. of (30) is convergent we can write

$$
\begin{aligned}
\left(\theta_{R}^{\mu \nu}, \varphi\right)= & \lim _{\epsilon \rightarrow 0}\left(\int d^{4} x Y(\rho-\epsilon) \Theta_{R}^{\mu \nu}(x) \varphi(x)\right. \\
& -\int d^{4} x Y(\rho-\epsilon) Y(L-\rho) \Theta_{R}^{\mu \nu}(x) \\
& \left.\times\left[\varphi(z)+\rho k^{\alpha} \partial_{\alpha} \varphi(z)\right]\right),
\end{aligned}
$$

which after a short calculation leads to

$$
\begin{aligned}
\theta_{R}^{\mu \nu}= & \hat{\theta}_{R}^{\mu \nu}-\int d \tau\left(\left[V^{\mu \nu}-\dot{U}^{\mu \nu}\right] \delta(x-z(\tau))\right. \\
& \left.-U^{\lambda \mu \nu} \partial_{\lambda} \delta(x-z(\tau))\right),
\end{aligned}
$$

where

$$
\begin{aligned}
\hat{\theta}_{R}^{\mu \nu}= & \lim _{\epsilon \rightarrow 0}\left[\Theta_{R}^{\mu \nu}(x) Y(\rho-\epsilon)\right. \\
& \left.-\frac{e^{2}}{\epsilon} \int d \tau\left(\frac{1}{2} v^{\mu} v^{\nu}+\frac{1}{6} \hat{\eta}^{\mu \nu}\right) \delta(x-z)\right]
\end{aligned}
$$

and the coefficients $V^{\mu \nu}, U^{\mu \nu}$, and $U^{\lambda \mu \nu}$ depend on $\tau$ and on the length scale $L$ :

$$
\begin{aligned}
V^{\mu \nu}= & -\frac{e^{2}}{6 L}\left(3 v^{\mu} v^{\nu}+\hat{\eta}^{\mu \nu}\right) \\
& +\frac{2 e^{2} L}{15}\left(5 a^{2} v^{\mu} v^{\nu}+2 a^{2} \hat{\eta}^{\mu \nu}-a^{\mu} a^{\nu}\right),
\end{aligned}
$$




$$
\begin{aligned}
U^{\mu \nu}= & \frac{2}{3} e^{2} L\left(a^{\mu} v^{\nu}+a^{\nu} v^{\mu}\right) \\
& +\frac{e^{2} L^{2}}{15}\left(5 a^{2} v^{\mu} v^{\nu}+2 a^{2} \hat{\eta}^{\mu \nu}-a^{\mu} a^{\nu}\right), \\
U^{\lambda \mu \nu}= & \frac{e^{2} L}{15}\left(3 a^{\mu} \hat{\eta}^{\lambda \nu}+3 a^{\nu} \hat{\eta}^{\lambda \mu}-2 a^{\lambda} \hat{\eta}^{\mu \nu}\right)+\frac{e^{2} L^{2}}{15} \\
& \times\left(2 a^{2}\left[v^{\mu} \hat{\eta}^{\lambda \nu}+v^{\nu} \hat{\eta}^{\lambda \mu}\right]-a^{\lambda}\left[a^{\mu} v^{\nu}+a^{\nu} v^{\mu}\right]\right) .
\end{aligned}
$$

We shall hereafter write

$$
\theta^{\mu \nu}=\theta_{R}^{\mu \nu}+\theta_{\mathrm{ext}}^{\mu \nu}+\theta_{\mathrm{mix}}^{\mu \nu} .
$$

Notice that $\theta^{\mu \nu} \in \mathcal{D}^{\prime}\left(\mathbb{R}^{4}\right) \subset \mathcal{D}^{\prime}\left(\mathbb{R}^{4}-\Gamma\right)$. Now, since $\Theta^{\mu \nu}(x)$ is locally summable in $\mathbb{R}^{4}-\Gamma$, it can be also considered as a generalized function $\Theta^{\mu \nu} \in \mathcal{D}^{\prime}\left(\mathbb{R}^{4}-\Gamma\right)$ and, as a consequence of (31), we have that

$$
\theta^{\mu \nu}=\Theta^{\mu \nu} \quad \text { in } \quad \mathcal{D}^{\prime}\left(\mathbb{R}^{4}-\Gamma\right) .
$$

\section{The total energy-momentum tensor}

The total energy-momentum tensor $t^{\mu \nu}$ is defined by the limit (21). As a consequence of (22) we have that, for any test function $\varphi \in \mathcal{D}\left(\mathbb{R}^{4}-\Gamma\right)$,

$$
\left(t^{\mu \nu}, \varphi\right)=\lim _{\epsilon \rightarrow 0} \int d^{4} x \Theta^{\mu \nu}(\epsilon, x) \varphi(x)
$$

and, using (27), (31), and (37), we obtain

$$
\left(t^{\mu \nu}, \varphi\right)=\left(\theta^{\mu \nu}, \varphi\right), \quad \forall \varphi \in \mathcal{D}\left(\mathbb{R}^{4}-\Gamma\right) .
$$

Therefore, $t^{\mu \nu}-\theta^{\mu \nu} \in \mathcal{D}^{\prime}\left(\mathbb{R}^{4}\right)$ has support on $\Gamma$ and, according to a well-known result [29], it can be written as a finite sum:

$$
\begin{aligned}
t^{\mu \nu}-\theta^{\mu \nu}= & \int d \tau\left[m^{\mu \nu}(\tau) \delta(x-z(\tau))\right. \\
& +m^{\alpha \mu \nu}(\tau) \partial_{\alpha} \delta(x-z(\tau))+\ldots \\
& \left.+m^{\alpha_{1} \ldots \alpha_{n} \mu \nu}(\tau) \partial_{\alpha_{1} \ldots \alpha_{n}} \delta(x-z(\tau))\right],
\end{aligned}
$$

where $m^{\left(\alpha_{1} \ldots \alpha_{r}\right) \mu \nu} v_{\alpha_{1}}=0, r=1 \ldots n$.

Combining now (38) with (32) and (37), we can write

$$
t^{\mu \nu}=\hat{\theta}_{R}^{\mu \nu}+\theta_{\mathrm{ext}}^{\mu \nu}+\theta_{\mathrm{mix}}^{\mu \nu}+t_{s}^{\mu \nu},
$$

with

$$
\begin{aligned}
t_{s}^{\mu \nu}:= & \int d \tau\left[p^{\mu \nu}(\tau) \delta(x-z(\tau))+p^{\alpha \mu \nu}(\tau) \partial_{\alpha} \delta(x-z(\tau))\right. \\
& \left.+\ldots+p^{\alpha_{1} \ldots \alpha_{n} \mu \nu}(\tau) \partial_{\alpha_{1} \ldots \alpha_{n}} \delta(x-z(\tau))\right],
\end{aligned}
$$

and

$$
\begin{gathered}
p^{\mu \nu}=m^{\mu \nu}+\dot{U}^{\mu \nu}-V^{\mu \nu}, \quad p^{\alpha \mu \nu}=m^{\lambda \mu \nu}+U^{\lambda \mu \nu}, \\
p^{\alpha_{1} \ldots \alpha_{n} \mu \nu}=m^{\alpha_{1} \ldots \alpha_{n} \mu \nu}, \quad n \geq 2,
\end{gathered}
$$

where $p^{\lambda \mu \nu} v_{\lambda}=0$, as it obviously follows from (36) and (38).

Notice that, although $t_{s}^{\mu \nu}$ is the only term in (39) whose support is the particle world line $\Gamma$, it is not to be interpreted as the "matter contribution" to the energymomentum tensor. For two reasons: first, the splitting $T^{\mu \nu}(\epsilon, x)=K^{\mu \nu}(\epsilon, x)+\Theta^{\mu \nu}(\epsilon, x)$ is not preserved in the point limit because neither $K^{\mu \nu}(\epsilon, x)$ nor $\Theta^{\mu \nu}(\epsilon, x)$ have a limit in $\mathcal{D}^{\prime}\left(\mathbb{R}^{4}\right)$ and, second, the other terms in (39) only describe a finite part of the "electromagnetic contribution" to the energy-momentum tensor, which results after removing infinities. So to speak, $t_{s}^{\mu \nu}$ can be seen as what is left after the infinities of opposite sign in the point limits of $K^{\mu \nu}(\epsilon, x)$ and $\Theta^{\mu \nu}(\epsilon, x)$ cancel each other. Therefore, we are not obliged to assign to this difference the value $m_{0} \int d \tau v^{\mu} v^{\nu} \delta(x-z(\tau))$, as is done in Ref. [3].

At this point we need to introduce an additional assumption stating the elementarity of the point charge. This consists of retaining as few terms in (40) as possible, in a way that no extra assumptions slip into the reasoning. We shall assume that $p^{\alpha_{1} \ldots \alpha_{n} \mu \nu}(\tau)=0$ for $n \geq 2$ and keep only the monopole and dipole terms,

$t_{s}^{\mu \nu}=\int d \tau\left[p^{\mu \nu}(\tau) \delta(x-z(\tau))+p^{\alpha \mu \nu}(\tau) \partial_{\alpha} \delta(x-z(\tau))\right]$.

It could seem that the minimal choice is taking $p^{\alpha \mu \nu}=$ 0 too, which unavoidably leads to the Lorentz-Dirac equation. Notice however that this choice actually implies an extra phenomenological assumption, namely, that the dipole term $U^{\alpha \mu \nu}$ in (32) - which depends on the arbitrary scale $L$ in the regularization process - exactly balances the dipole term $m^{\alpha \mu \nu}$ in (38). We shall hence leave $p^{\alpha \mu \nu} \neq 0$ in spite that [1] " . . .one would hardly expect [it] to apply to a simple thing as an electron", because the preacceleration or runaways associated to the Lorentz-Dirac equation seem still harder to accept.

\section{CONSERVATION LAWS AND EQUATIONS OF MOTION}

The local conservation laws (10) will then yield some restrictions on the coefficients $p^{\mu \nu}$ and $p^{\lambda \mu \nu}$ [30,31]. From the symmetry of $t^{\mu \nu}$ it follows that $p^{\mu \nu}=p^{\nu \mu}, p^{\lambda \mu \nu}=$ $p^{\lambda \nu \mu}$. Then, it is helpful to separate these coefficients in their components, respectively, parallel and orthogonal to the velocity $v^{\mu}$ :

$$
\left.\begin{array}{c}
p^{\mu \nu}=M v^{\mu} v^{\nu}+p^{\mu} v^{\nu}+p^{\nu} v^{\mu}+p_{1}^{\mu \nu}, \\
p^{\lambda \mu \nu}=Q^{\lambda} \boldsymbol{v}^{\mu} \boldsymbol{v}^{\nu}+Q^{\lambda \mu} \boldsymbol{v}^{\nu}+Q^{\lambda \nu} \boldsymbol{v}^{\mu}+Q^{\lambda \mu \nu},
\end{array}\right\}
$$

where all tensors and vectors other than $v^{\mu}$ are orthogonal to the velocity. The local conservation law (10) then implies that

$$
\partial_{\mu} \hat{\theta}_{R}^{\mu \nu}+\partial_{\mu} \theta_{\mathrm{ext}}^{\mu \nu}+\partial_{\mu} \theta_{\mathrm{mix}}^{\mu \nu}+\partial_{\mu} t_{s}^{\mu \nu}=0 .
$$


Now, since $\theta_{\mathrm{ext}}^{\mu \nu}$ is the energy-momentum tensor of a free electromagnetic field, $\partial_{\mu} \theta_{\mathrm{ext}}^{\mu \nu}=0$. Similarly, the cross term contribution is

$$
\partial_{\mu} \theta_{\mathrm{mix}}^{\mu \nu}=-F_{\mathrm{ext}}^{\nu \mu} j_{\mu}=-e \int d \tau F_{\mathrm{ext}}^{\nu \mu}(z) v_{\mu}(\tau) \delta(x-z(\tau))
$$

and (see Appendix A [Eq. (A4)] for details)

$$
\partial_{\mu} \hat{\theta}_{R}^{\mu \nu}=\frac{2}{3} e^{2} \int d \tau\left[a^{2} v^{\nu}-\dot{a}^{\nu}\right] \delta(x-z(\tau)) .
$$

Finally, using (42) and integrating by parts, we also obtain

$$
\begin{aligned}
\partial_{\mu} t_{s}^{\mu \nu}= & \int d \tau\left[\frac{d}{d \tau}\left(M v^{\nu}+p^{\nu}+a_{\lambda}\left[Q^{\lambda} v^{\nu}+Q^{\lambda \nu}\right]\right) \delta(x-z)+\left(v^{\nu} p^{\mu}+p_{1}^{\mu \nu}+\hat{\eta}_{\lambda}^{\mu} \frac{d}{d \tau}\left[Q^{\lambda} v^{\nu}+Q^{\lambda \nu}\right]\right) \partial_{\mu} \delta(x-z)\right. \\
& \left.+\left(Q^{\lambda \mu \nu}+Q^{\lambda \mu} v^{\nu}\right) \partial_{\lambda \mu} \delta(x-z)\right]
\end{aligned}
$$

and, substituting (44)-(46) into (44), we arrive at

$$
\begin{aligned}
0= & \int d \tau\left[\left\{\frac{d}{d \tau}\left(M v^{\nu}+p^{\nu}+a_{\lambda}\left[Q^{\lambda} v^{\nu}+Q^{\lambda \nu}\right]\right)+\frac{2}{3} e^{2}\left(a^{2} v^{\nu}-\dot{a}^{\nu}\right)-F^{\nu}\right\} \delta(x-z)\right. \\
& \left.+\left(v^{\nu} p^{\mu}+p_{1}^{\mu \nu}+\hat{\eta}_{\lambda}^{\mu} \frac{d}{d \tau}\left[Q^{\lambda} v^{\nu}+Q^{\lambda \nu}\right]\right) \partial_{\mu} \delta(x-z)+\left(Q^{\lambda \mu \nu}+Q^{\lambda \mu} v^{\nu}\right) \partial_{\lambda \mu} \delta(x-z)\right],
\end{aligned}
$$

where $F^{\nu} \equiv e F_{\mathrm{ext}}^{\nu \mu}(z) v_{\mu}$.

As the derivatives of $\delta$-functions in the r.h.s. are contracted with tensors that are transversal to the world line, each term must vanish separately and therefore

$$
\frac{d}{d \tau}\left(M v^{\nu}+p^{\nu}+a_{\lambda}\left[Q^{\lambda} v^{\nu}+Q^{\lambda \nu}\right]\right)+\frac{2}{3} e^{2}\left(a^{2} v^{\nu}-\dot{a}^{\nu}\right)=F^{\nu},
$$

$$
\begin{gathered}
v^{\nu} p^{\mu}+p_{1}^{\mu \nu}+\hat{\eta}_{\lambda}^{\mu} \frac{d}{d \tau}\left[Q^{\lambda} v^{\nu}+Q^{\lambda \nu}\right]=0, \\
Q^{(\lambda \mu) \nu}+Q^{(\lambda \mu)} v^{\nu}=0 .
\end{gathered}
$$

Since $Q^{\lambda \mu}$ and $Q^{\lambda \mu \nu}$ are orthogonal to $v_{\lambda}$ and $Q^{\lambda \mu \nu}=$ $Q^{\lambda \nu \mu}$, Eq. (50) implies that

$$
Q^{(\lambda \mu)}=0 \text { and } Q^{\lambda \mu \nu}=0 .
$$

Substituting this into (49), we obtain

$$
\begin{gathered}
p^{\mu}=-\dot{Q}_{\perp}^{\mu}-Q^{\mu \lambda} a_{\lambda}, \\
p_{1}^{\mu \nu}=-Q^{\mu} a^{\nu}-\dot{Q}^{\mu \nu}+v^{\nu} Q^{\mu \lambda} a_{\lambda}+v^{\mu} Q^{\lambda \nu} a_{\lambda},
\end{gathered}
$$

where the subindex $\perp$ means the projection orthogonal to $v^{\mu}$. Since $p_{1}^{\mu \nu}$ is symmetric and $Q^{\mu \nu}$ is skewsymmetric, it follows that

$$
p_{1}^{(\mu \nu)}=-Q^{(\mu} a^{\nu)} \quad \text { and } \quad \dot{Q}_{\perp}^{\mu \nu}=-Q^{[\mu} a^{\nu]} .
$$

Finally, substituting (51), (52), and (54) into (48), after a short manipulation we arrive at

$$
\frac{d}{d \tau}\left([M+(Q a)] v^{\nu}-\dot{Q}_{\perp}^{\nu}-2 Q^{\nu \lambda} a_{\lambda}\right)-\frac{2}{3} e^{2} \dot{a}_{\perp}^{\nu}=F^{\nu} .
$$

On the basis of solely the conservations of total linear and angular momenta, plus an assumption (41) concerning the elementarity of the charge, we have thus found that:

(1) The quantities $M, Q^{\lambda}, \ldots, Q^{\lambda \mu \nu}$ in Eqs. (42) can be written in terms of some particle variables: $M, Q^{\lambda}$, and $Q^{[\lambda \mu]}$, which, owing to the symmetries and constraints, amount to only seven independent variables.

(2) These, together with the world line variables $z^{\mu}(\tau), v^{\mu}(\tau), \ldots$ are subject to the differential system (54) and (55).

Except for notation, Eqs. (54) and (55) coincide with a similar result obtained by Lozada - Eqs. (3.20) and (3.21) in Ref. [23] - by a pretty similar method. They are not the equations of motion yet. Indeed, owing to the symmetries, they amount to 7 ordinary differential equations for 10 unknowns, namely, $M, Q^{\lambda}, Q^{\mu \nu}$, and $v^{\nu}$.

The particle variables $M, Q^{\lambda}$, and $Q^{\mu \nu}$ are somewhat related to the (phenomenological) mass of the particle, its spin, and the shift of $z^{\nu}(\tau)$ from the "center-of-mass" world line. These relationships will be useful to find complementary conditions that help to delimit the final equation of motion. However, they are not obvious at all and are studied in the next section.

\section{A. Particle's linear and angular momenta}

In order to have a clue on the physical meaning of the variables $M, Q^{\lambda}$, and $Q^{\lambda \nu}$, and of Eqs. (54) and (55) we examine the linear and angular momenta of the point charge, i.e., those associated to the singular part $t_{s}^{\mu \nu}$ of the energy-momentum tensor (39).

Given an energy-momentum tensor $T^{\mu \nu}$, the linear and angular momenta contained in the hypersurface $\Sigma$ are [32], respectively, 


$$
\begin{aligned}
& P^{\mu}(\Sigma)=\int_{\Sigma} T^{\mu \nu}(x) d \Sigma_{\nu} \text { and } \\
& J^{\mu \nu}(\Sigma)=\int_{\Sigma} x^{\mu} \wedge T^{\nu \lambda}(x) d \Sigma_{\lambda},
\end{aligned}
$$

where $d \Sigma_{\nu}$ is the hypersurface element.

These definitions apply when $T^{\mu \nu}(x)$ are locally summable functions, but are useless for the generalized functions $t_{s}^{\mu \nu}$. Indeed, they imply integrating a generalized function with respect to some parameters that depend on the variables $x$ of the test functions acted by $t_{s}^{\mu \nu}$, and this is not a well-defined operation.

However, the above definitions can be taken as a starting point for an extension that applies to our case. For the sake of simplicity, we shall assume that $\Sigma$ is a hyperplane, with unit normal vector $w_{\mu}$, which is furthermore assumed to be timelike. Take a fixed point $z \in \Sigma$ and consider a constant orthonormal base, $e_{(1)}^{\mu}, e_{(2)}^{\mu}, e_{(3)}^{\mu}, e_{(4)}^{\mu}=w^{\mu}$, in spacetime, then take the coordinates:

$\lambda=\left(x^{\nu}-z^{\nu}\right) w_{\mu}, \quad X^{i}=\left(x_{\nu}-z_{\nu}\right) e_{(i)}^{\nu}, \quad i=1,2,3$.

$\Sigma$ is characterized by $\lambda=0$ and the hypersurface element is $d \Sigma_{\nu}=-d^{3} X w_{\nu}$.

Consider then a regularization of $\delta(\lambda)$, i.e., a test function $\omega_{\epsilon}(\lambda) \in \mathcal{D}(\mathbb{R})$ such that $\omega_{\epsilon}(\lambda) \rightarrow \delta(\lambda)$ in $\mathcal{D}^{\prime}$. Furthermore, take a second test function $\eta(\vec{X}) \in \mathcal{D}\left(\mathbb{R}^{3}\right)$ such that $\eta(\vec{X})=1$ for $|\vec{X}|<L$, for some given $L$. Definitions (56) can then be written as:

$$
\begin{aligned}
P^{\mu}(\Sigma) & =\lim _{\epsilon \rightarrow 0} \int_{\mathbb{R}^{4}} d^{4} x \omega_{\epsilon}(\lambda) \eta(\vec{X}) T^{\mu \nu}(x) w_{\nu}, \\
J^{\mu \nu}(\Sigma) & =\lim _{\epsilon \rightarrow 0} \int_{\mathbb{R}^{4}} d^{4} x \omega_{\epsilon}(\lambda) \eta(\vec{X}) x^{\mu} \wedge T^{\nu \rho}(x) w_{\rho},
\end{aligned}
$$

and, since $\omega_{\epsilon}(\lambda) \eta(\vec{X})$ is a test function in $\mathcal{D}\left(\mathbb{R}^{4}\right)$, these formulae are easily extended to the case that $T^{\mu \nu}$ are the generalized functions $t_{s}^{\mu \nu}$

$$
\begin{aligned}
P_{s}^{\mu}(\Sigma) & =\lim _{\epsilon \rightarrow 0}\left(t_{s}^{\mu \nu} w_{\nu}, \omega_{\epsilon}(\lambda) \eta(\vec{X})\right), \\
J_{s}^{\mu \nu}(\Sigma) & =\lim _{\epsilon \rightarrow 0}\left(x^{\mu} \wedge t_{s}^{\nu \rho} w_{\rho}, \omega_{\epsilon}(\lambda) \eta(\vec{X})\right) .
\end{aligned}
$$

Since the spacelike hyperplane $\Sigma$ is unambiguously determined by $z^{\mu}(\tau)$ and $w^{\mu}$, i.e., its intersection with the particle's world line and the unit normal vector, we shall hereafter write $P_{s}^{\mu}(\tau, w)$ and $J_{s}^{\mu \nu}(\tau, w)$ instead of $P_{s}^{\mu}(\Sigma)$ and $J_{s}^{\mu \nu}(\Sigma)$, respectively.

If $\epsilon$ is small enough, we have that

$$
\begin{aligned}
& {\left[\omega_{\epsilon}(\lambda) \eta(\vec{X})\right]_{z(\tilde{\tau})}=\omega_{\epsilon}(\lambda(\tilde{\tau})) \quad \text { and }} \\
& \quad\left(\partial_{\rho}\left[\omega_{\epsilon}(\lambda) \eta(\vec{X})\right]\right)_{z(\tilde{\tau})}=\omega_{\epsilon}(\lambda(\tilde{\tau})) w_{\rho},
\end{aligned}
$$

where $\lambda(\tilde{\tau}) \equiv\left[z^{\nu}(\tilde{\tau})-z^{\nu}(\tau)\right] w_{\nu}$ has been taken and $\partial_{\rho} \lambda=w_{\rho}$ has been used. Substituting now (41) into (57), we have that

$$
\begin{aligned}
P_{S}^{\mu}(\tau, w)= & \lim _{\epsilon \rightarrow 0} \int d \tilde{\tau}\left[-p^{\mu \nu}(\tilde{\tau}) w_{\nu} \omega_{\epsilon}(\lambda(\tilde{\tau}))\right. \\
& \left.+p^{\rho \mu \nu}(\tilde{\tau}) w_{\rho} w_{\nu} \omega_{\epsilon}^{\prime}(\lambda(\tilde{\tau}))\right]
\end{aligned}
$$

which is easily evaluated by changing the variable to $\lambda$ and recalling that $\omega_{\epsilon}(\lambda) \rightarrow \delta(\lambda)$. We also obtain

$$
\begin{aligned}
P_{s}^{\mu}(\tau, w)= & \frac{p^{\mu \nu}(\tau) w_{\nu}}{w^{\alpha} v_{\alpha}(\tau)}+\frac{\dot{p}^{\rho \mu \nu}(\tau) w_{\rho} w_{\nu}}{\left[w^{\alpha} v_{\alpha}(\tau)\right]^{2}} \\
& -\frac{w^{\beta} a_{\beta}(\tau) p^{\rho \mu \nu}(\tau) w_{\rho} w_{\nu}}{\left[w^{\alpha} v_{\alpha}(\tau)\right]^{3}} .
\end{aligned}
$$

Taking then $P_{s}^{\mu}(\tau) \equiv P_{s}^{\mu}(\tau, v(\tau))$ as the particle linear momentum and including (42), we have that

$$
P_{s}^{\mu}(\tau)=[M+(Q a)] v^{\mu}-\dot{Q}_{\perp}^{\mu}-2 Q^{\mu \rho} a_{\rho}
$$

and Eq. (55) reads

$$
\frac{d P_{s}^{\nu}}{d \tau}=F^{\nu}+\frac{2}{3} e^{2} \dot{a}_{\perp}^{\nu}
$$

On its turn, it follows from (57) that angular momentum can be separated as $J_{s}^{\mu \nu}(\tau, w)=z^{\mu}(\tau) \wedge P_{s}^{\nu}(\tau, w)+$ $\sum_{s}^{\mu \nu}(\tau, w)$, where

$$
\Sigma_{s}^{\mu \nu}(\tau, w) \equiv \lim _{\epsilon \rightarrow 0}\left(\left[x^{\mu}-z^{\mu}(\tau)\right] \wedge t_{s}^{\nu \rho} w_{\rho}, \omega_{\epsilon}(\lambda) \eta(\vec{X})\right) .
$$

By a short calculation we readily obtain that: $\Sigma^{\mu \nu}\left(\tau_{0}, w\right)=$ $2 p^{[\mu \nu] \rho}\left(\tau_{0}\right) w_{\rho}$ and, defining $\Sigma^{\mu \nu}(\tau) \equiv \Sigma^{\mu \nu}(\tau, v(\tau))$ as above, we have that:

$$
\Sigma_{s}^{\mu \nu}(\tau)=-2 Q^{[\mu \nu]}-Q^{\mu} \wedge v^{\nu} .
$$

Then Eq. (54) reads

$$
\left(\frac{d \Sigma_{s}^{\mu \nu}}{d \tau}\right)_{\perp}=0
$$

\section{B. Spinless particles}

To model a spinless particle we require that the PauliLubanski vector [33] vanishes. This amounts to $J_{s}^{\mu \nu} \wedge$ $P_{s}^{\mu}=0$ or, equivalently,

$$
\Sigma_{s}^{\mu \nu}=L^{\mu} \wedge P_{s}^{\nu} \quad \text { for some vector } L^{\mu} .
$$

Since $L^{\mu}$ is determined up to a multiple of $P_{s}^{\mu}$, it can be chosen so that $L^{\mu} v_{\mu}=0$. Then, projecting $\Sigma_{s}^{\mu \nu}$ onto $v_{\nu}$ and including (58) and (59), we obtain: $Q^{\mu}=-[M+$ $(Q a)] L^{\mu}$ or, equivalently,

$$
Q^{\mu}=-\frac{M}{1+(L a)} L^{\mu}, \quad \text { and } \quad M+(Q a)=\frac{M}{1+(L a)} .
$$

Introducing this again in (59) and (61), including (58), we 
have that:

$$
Q^{\mu \nu}=-\frac{M}{2[1+(L a)]^{2}} L^{\mu} \wedge \dot{L}_{\perp}^{\nu} .
$$

Substituting this and (62) in (58), the linear momentum of a spinless charge is

$$
P_{s}^{\mu}=\frac{M}{1+(L a)} v^{\mu}+\frac{M}{[1+(L a)]^{2}} \dot{L}_{\perp}^{\mu}
$$

and, as a consequence of (63), angular momentum can be written as:

$$
J_{s}^{\mu \nu}=Z^{\mu} \wedge P_{s}^{\nu}, \quad \text { with } \quad Z^{\mu}=z^{\mu}+L^{\mu},
$$

i.e., a purely orbital angular momentum for the "center-ofmass" point $Z^{\mu}(\tau)$. Moreover, substituting $\dot{L}_{\perp}^{\mu}=$ $\dot{L}^{\mu}-(L a) v^{\mu}$ in Eq. (64), we have that:

$$
P_{s}^{\mu}=\frac{M}{[1+(L a)]^{2}}\left(v^{\mu}+\dot{L}^{\mu}\right)=\frac{M}{[1+(L a)]^{2}} \frac{d Z^{\mu}}{d \tau},
$$

which is the familiar relation between linear momentum and the center-of-mass velocity. The proper mass is

$$
m_{s}:=\sqrt{-P_{s}^{\mu} P_{s \mu}}=\frac{M}{[1+(L a)]^{2}} \sqrt{[1+(L a)]^{2}-\dot{L}_{\perp}^{2}} .
$$

(Notice that $\tau$ is not the center-of-mass proper time.)

Furthermore, combining (60) and (63), we obtain that $L^{\mu} \wedge \dot{P}_{s}^{\nu}=0$, which means that $L^{\mu}$ is proportional to $\dot{P}_{s}^{\mu}$, that is,

$$
L^{\mu}=l\left(F^{\mu}+\frac{2}{3} e^{2} \dot{a}_{\perp}^{\mu}\right), \quad \text { for some scalar function } l(\tau) .
$$

Finally, substituting (62) and (63) in (42) and including Eqs. (51)-(54), we obtain

$$
\begin{aligned}
p^{\lambda \mu \nu}= & -\frac{M}{[1+(L a)]^{2}}\left(L^{\lambda} v^{\mu} v^{\nu}+L^{\lambda} \dot{L}^{(\mu} \boldsymbol{v}^{\nu)}\right. \\
& \left.-\dot{L}_{\perp}^{\lambda} L^{(\mu} \boldsymbol{v}^{\nu)}\right), \\
p^{\mu \nu}= & \frac{M}{[1+(L a)]^{2}}\left(v^{\mu} \boldsymbol{v}^{\nu}+\dot{L}^{(\mu} \boldsymbol{v}^{\nu)}\right)+\frac{d}{d \tau} \\
& \times\left(\frac{M}{1+(L a)} L^{(\mu} \boldsymbol{v}^{\nu)}\right) .
\end{aligned}
$$

\section{Summary}

A classical spinless point charge is therefore described by

(1) the electric current density $j^{\mu}=e \int d \tau v^{\mu}(\tau) \delta(x-$ $z(\tau))$, where the electric charge $e$ is a constant scalar, and

(2) the total energy-momentum tensor $t^{\mu \nu}=$ $t_{s}^{\mu \nu}+\hat{\theta}_{R}^{\mu \nu}+\theta_{\mathrm{ext}}^{\mu \nu}+\theta_{\mathrm{mix}}^{\mu \nu}$, where $\hat{\theta}_{R}^{\mu \nu}$ and $t_{s}^{\mu \nu}$ are, respectively, given by (33) and (41).

(3) As a consequence of linear and angular momenta conservation, supplemented with the elementarity hypothesis and the spinless condition, the coefficients $p^{\lambda \mu \nu}$ and $p^{\mu \nu}$ occurring in $t_{s}^{\mu \nu}$ can be written as (66) and (67) in terms of some matter variables, namely $M$ and $L^{\nu}$ plus the world line variables $z^{\mu}(\tau), v^{\nu}(\tau), \ldots$, which are subject to the conditions

$$
\frac{d}{d \tau}\left[\frac{M}{[1+(L a)]^{2}}\left(v^{\mu}+\dot{L}^{\mu}\right)\right]=F^{\mu}+\frac{2}{3} e^{2} \dot{a}_{\perp}^{\mu},
$$

with

$$
L^{\mu}=l\left(F^{\mu}+\frac{2}{3} e^{2} \dot{a}_{\perp}^{\mu}\right),
$$

for some scalar function $l(\tau)$.

These two equations have been derived on the only basis that linear and angular momenta are conserved, supplemented with an assumption of elementarity plus the condition that the particle does not have spin. Notice that (68) is less restrictive than the Lorentz-Dirac equation, which corresponds to taking $L^{\mu}=0$ (or $l=0$ ).

\section{THE EQUATION OF MOTION}

Equation (68) supplemented with (69) is not yet the law of motion. Indeed, it consists of four equations for five unknowns, namely, $M, l$, and $z^{\mu}$ (due to the constraint $v^{\mu} v_{\mu}=-1, z^{\nu}$ are only three independent variables). The motion of the particle is therefore underdetermined.

This should not be surprising. The problem in dynamics of continuous media for $\epsilon>0$, as we have posed it (i.e., the local conservation laws for momentum and angular momentum: $\partial_{\mu} T^{\mu \nu}=0$ and $T^{\mu \nu}=T^{\nu \mu}$ ) is itself underdetermined until constitutive and state equations for the material sustaining the electric charge are provided. (In other approaches to the dynamics of a point charge, e.g. $[8,12,34]$, the role of constitutive equations is played by the assumption that the charge is rigidly distributed.)

Constitutive and state equations are relations among the components of $K^{\mu \nu}$, the material part of the energymomentum tensor. Since $t_{s}^{\mu \nu}$ is the singular part of the limit, $\epsilon \rightarrow 0$, of $T^{\mu \nu}=K^{\mu \nu}+\Theta^{\mu \nu}$, any constitutive equation that we assume on $K^{\mu \nu}$ will yield, in the limit $\epsilon \rightarrow 0$, a constitutive relation connecting the tensors $p^{\mu \nu}$ and $p^{\lambda \mu \nu}$ and maybe the world line variables $a^{\mu}, \dot{a}^{\mu}, \ldots$ (particularly, the assumption of a rigid spherical shell in [8] or a rigid dumbell [34] both lead to the constitutive relation $l=0)$.

Therefore, instead of advancing such a constitutive equation for the continuous distribution of matter and charge for $\epsilon>0$, then reexamining the problem and taking the limit $\epsilon \rightarrow 0$ to determine a final equation of motion, we can proceed by directly posing a constitutive relation con- 
necting the scalars $M, l$ and the world line invariants (proper acceleration, curvature, and torsion).

Notice that, although it is the simplest choice and looks suitable for an elementary charge, a prescription like $l=0$ is not an appropriate constitutive relation, because for this choice (65) becomes the Lorentz-Dirac equation whose solutions are either preaccelerated or runaway, as commented in the introduction. Furthermore, even though the prescription $l=0$ is also supported by the above mentioned models based on a rigid charge distribution, the assumption of rigidity carries the violation of causality with itself. Indeed, from a kinematical viewpoint rigid models are not necessarily in conflict with relativity, but from a dynamical viewpoint the assumption of a rigid distribution of matter implies that, in order to keep the distribution's shape, stresses transmitting instantaneously are needed, so violating causality.

In our view, any guess for a constitutive relation should observe the following requirements:

(1) it connects $M, l, a^{\nu}$, and maybe some of their derivatives,

(2) when $a^{\nu}, l$ and also all their derivatives vanish, then $M=m_{0}$, a constant, and

(3) if the point charge is acted by an external force $F^{\nu}$ that vanishes for $\tau<0$ and for $\tau>\tau_{1}$, then

(i) $a^{\nu}(\tau)=0, M(\tau)=m_{0}$, and $L^{\nu}(\tau)=0$ for $\tau<0$ and

(ii) $a^{\nu} \rightarrow 0, M \rightarrow m_{0}$, and $L^{\nu} \rightarrow 0$ asymptotically in the future.

(The proper mass is assigned the same value $m_{0}$ in both infinite past and future, because we are assuming that the particle's "identity" is preserved.)

In what follows we shall try to illustrate that there exist constitutive relations compatible with these three conditions, regardless of their underlying physical foundations, e.g. classical continuum mechanics, QED, or whatsoever. In particular, we shall examine the case of accelerated rectilinear motion. (Recall that the Lorentz-Dirac equation presents noncausal behavior even in this simple case.)

\section{A. Rectilinear motion}

Consider a point charge that initially is unaccelerated and free. Then, during the interval $0 \leq \tau \leq \tau_{1}$, it is acted by an external force in a constant direction along the $X^{1}$ axis. The charge world line will remain in the plane $X^{1} X^{4}$ in spacetime and therefore,

$$
\frac{d v^{\mu}}{d \tau}=a \hat{a}^{\mu} \quad \text { and } \quad \frac{d a^{\mu}}{d \tau}=\dot{a} \hat{a}^{\mu}+a^{2} v^{\mu},
$$

where $\hat{a}^{\mu}$ is the unit vector parallel to $a^{\mu}$, i.e., the first normal to the world line. Moreover, $F^{\mu}=F \hat{a}^{\mu}$ and, by (69),

$$
\begin{aligned}
L^{\mu} & =\tilde{l} \hat{a}^{\mu}, \\
\dot{L}^{\mu} & =\tilde{\tilde{l}} \hat{a}^{\mu}+a \tilde{l} \hat{a}^{\mu}, \quad \text { with } \quad \tilde{l} \equiv l\left(F+\frac{2}{3} e^{2} \dot{a}\right) .
\end{aligned}
$$

In this case, the only nonvanishing components of Eq. (68) are

$$
\begin{aligned}
& \left(\| v^{\mu}\right) \quad \frac{d}{d \tau}\left(\frac{M}{1+\tilde{l} a}\right)+\frac{M \dot{\tilde{l}} a}{(1+\tilde{l} a)^{2}}=0 \\
& \left(\perp v^{\mu}\right) \quad \frac{M a}{1+\tilde{l} a}+\frac{d}{d \tau}\left(\frac{M \dot{\tilde{l}}}{(1+\tilde{l} a)^{2}}\right)=F+\frac{2}{3} e^{2} \dot{a},
\end{aligned}
$$

which introducing the new variable $q(a, l, \dot{a})=-\frac{M \tilde{l}}{1+\tilde{l} a}$, become

$$
\begin{gathered}
\left(\| v^{\mu}\right) \quad \frac{d}{d \tau}(M+q a)=a \dot{q}, \\
\left(\perp v^{\mu}\right) \quad a(M+q a)-\ddot{q}-\frac{2}{3} e^{2} \dot{a}=F .
\end{gathered}
$$

These two equations must be supplemented with a constitutive relation $M=M(a, q, \dot{q})$ in order that evolution is determined. The phase space is therefore coordinated by $(a, q, \dot{q})$.

We would expect that while the charge is not acted by any force, $F(\tau)=0,-\infty<\tau<0$, then it remains in a state of uniform rectilinear motion and the energymomentum tensor is the one corresponding to a free particle together with its Coulomb field, i.e., Eqs. (39), (66), and (67) with $L^{\mu}=0$ and $\dot{L}^{\mu}=0$ :

$$
\begin{array}{cl}
a(\tau)=0, \quad & M(\tau)=m_{0}, \quad q(\tau)=\dot{q}(\tau)=0, \\
& -\infty<\tau<0 .
\end{array}
$$

If an external force is then switched on, $F(\tau) \neq 0,0 \leq$ $\tau<\tau_{1}$, then $a, M, q$, and $\dot{q}$ evolve according to (70) and (71) with the initial data inferred from (72) and the continuity of the orbit in phase space. This determines

$a(\tau), \quad M(\tau), \quad q(\tau), \quad$ and $\quad \dot{q}(\tau)$ for $0<\tau<\tau_{1}$.

Next the particle is not acted by a force anymore and what we would expect is that it asymptotically tends towards a free state, i.e.,

$$
\begin{gathered}
a(\tau) \rightarrow 0, \quad M(\tau) \rightarrow m_{0}, \quad q(\tau) \rightarrow 0, \\
\dot{q}(\tau) \rightarrow 0 \quad \text { for } \tau \rightarrow \infty
\end{gathered}
$$

(with the same asymptotical value $m_{0}$ for the mass, in order that the particle's identity is preserved).

A way to achieve this behavior consists in that the dynamical system (70) and (71) supplemented with the constitutive relation has only one asymptotically stable equilibrium point for $a=q=\dot{q}=0$ and $M(0,0,0)=m_{0}$.

\section{B. A dynamical system}

Using the constant $\tau_{0} \equiv \frac{2 e^{2}}{3 m_{0}}$, we introduce the new dimensionless variables 


$$
\begin{gathered}
t \equiv \frac{\tau}{\tau_{0}}, \quad 1+\mu \equiv \frac{M+q a}{m_{0}}, \\
\alpha \equiv a \tau_{0}, \quad \rho \equiv \frac{q}{m_{0} \tau_{0}}
\end{gathered}
$$

and reduce (70) and (71) with $F=0$ to the simpler equivalent system

$$
\begin{gathered}
\mu^{\prime}=a \rho^{\prime}, \quad \rho^{\prime \prime}+\alpha^{\prime}=\alpha(1+\mu), \\
\mu=\mu\left(\alpha, \rho, \rho^{\prime}\right),
\end{gathered}
$$

where "prime" means "derivative with respect to $t$."

Then, by differentiating the constitutive relation and introducing the variable $x \equiv \rho^{\prime}+\alpha$, we obtain

$$
\left.\begin{array}{c}
\rho^{\prime}=x-\alpha, \\
x^{\prime}=\alpha(1+\mu), \\
\alpha^{\prime}=A(\alpha, \rho, x),
\end{array}\right\}
$$

where

$$
A(\alpha, \rho, x) \equiv \frac{1}{\mu_{\alpha}}\left[(x-\alpha)\left(\alpha-\mu_{\rho}\right)-\alpha \mu_{x}(1+\mu)\right] .
$$

This dynamical system is already in normal form and is defined in the entire phase space provided that the function $A(\alpha, \rho, x)$ has no singularities. Particularly, if we choose $\mu$ so that it is a solution of

$$
A_{0}(\alpha, \rho, x) \mu_{\alpha}+(x-\alpha) \mu_{\rho}+\alpha(1+\mu) \mu_{x}=\alpha(x-\alpha)
$$

with $\mu(0,0,0)=0, A_{0}(\alpha, \rho, x)=l \alpha+p \rho+r x$, and $l, p$, $r$ constants, then the dynamical system (77) becomes

$$
\frac{d}{d t}\left(\begin{array}{l}
\alpha \\
\rho \\
x
\end{array}\right)=\left(\begin{array}{ccc}
l & p & r \\
-1 & 0 & 1 \\
1 & 0 & 0
\end{array}\right)\left(\begin{array}{l}
\alpha \\
\rho \\
x
\end{array}\right)+\left(\begin{array}{c}
0 \\
0 \\
\mu \alpha
\end{array}\right) .
$$

If $p \neq 0$, the equilibrium points are

$$
\begin{aligned}
& P_{\mathrm{I}}: \alpha=\rho=x=0, \quad P_{\mathrm{II}}: x=\alpha=\alpha_{0}, \\
& \rho_{0}=-\frac{l+r}{p} \alpha_{0}, \quad \text { and } \quad \mu\left(\alpha_{0}, \rho_{0}, \alpha_{0}\right)=-1 .
\end{aligned}
$$

Moreover, the constants $l, p$, and $r$ can be chosen so that the characteristic equation at $P_{\mathrm{I}}$,

$$
X^{3}-l X^{2}+(p-r) X-p=0,
$$

has three negative solutions and hence $P_{\mathrm{I}}$ is an asymptotically stable equilibrium point.

In Appendix B [Eq. (B4)] we see how a solution $\mu=$ $\mu(\alpha, \rho, x)$ of Eq. (76) that vanishes at $P_{\mathrm{I}}=(0,0,0)$ can be perturbatively obtained and is valid at least in a neighborhood of this phase point.

Now, (74) can be used to obtain the constitutive equation

$$
M=m_{0}-q a m_{0} \mu\left(a \tau_{0}, \frac{q}{m_{0} \tau_{0}}, a \tau_{0}+\frac{\dot{q}}{m_{0}}\right) .
$$

This, together with Eqs. (70) and (71), determines a motion of the charge that is free of both preacceleration and runaways, provided that the force $F$ acts only during a finite interval of time. Indeed, if the charge is unaccelerated in past infinity it remains so until its state is altered because $F$ has started to act. Then, when the force ceases, the charge tends to the asymptotically stable equilibrium point $a=0$, $q=\dot{q}=0$, at least if the system was close enough when the force dissappeared.

\section{CONCLUSION}

By studying the energy-momentum balance of a classical point charge with the electromagnetic field, we have obtained that

(1) the total energy-momentum tensor consists of (i) a regular part, which comes from the external field contribution plus the regularization of the self-field contribution, and (ii) a singular part, with support on the charge world line.

(2) This singular part depends on the matter variables $M, Q^{\mu}$, and $Q^{\mu \nu}$ and on world line variables $v^{\mu}(\tau), a^{\mu}(\tau), \ldots$, which are constrained to fulfill Eqs. (54) and (55).

These equations are similar to those derived in Ref. [23] and for $Q^{\mu \nu}=0$ reduce to the one derived by Honig and Szamosi [25]. The Lorentz-Dirac equation is obtained only if the constitutive relation $Q=0$ is set by hand. The wellknown troubles that suffers the Lorentz-Dirac equation are due to this bad choice rather than to energy-momentum conservation itself.

Then, instead of taking $Q^{\mu \nu}=0$ as the characterization of a spinless charge, we have required the vanishing of the Pauli-Lubanski vector. This implies that all matter variables depend on two scalars, namely $M$ and $l$-Eqs. (62), (63), and (69) - and on kinematical quantities. All these variables are subject to Eq. (65).

As the number of equations is less than the number of degrees of freedom, the law of motion of a spinless charge is not unambiguously determined by conservation of linear and angular momenta. To achieve a unique equation of motion, a constitutive relation connecting $M, l$, and the world line variables must be added by hand. (This constitutive relation would be a sort of remnant, after the limit $\epsilon \rightarrow 0$, of the constitutive and state equations which are needed in fluid mechanics as a supplement to conservation laws.)

Finally, as an illustration and without intending a physical meaning, we have then seen that, at least for rectilinear motion, a constitutive relation $M=M(a, Q, \dot{Q})$ can be found such that, combined with Eq. (65) leads to an equation of motion for the point charge that is free from both preacceleration and runaways. That is, if a charge with proper mass $m_{0}$ is initially at rest and then is acted by an external force which lasts only a finite interval of time, then there is no acceleration before the force starts and, 
when its action ceases, the motion tends asymptotically to be rectilinear uniform and the proper mass tends to $m_{0}$.

\section{ACKNOWLEDGMENTS}

The work of J. Ll. and A. M. is supported by Ministerio de Ciencia y Tecnología, BFM2003-07076, and Generalitat de Catalunya, 2001SGR-00061 (DURSI). J.M.A. was supported by the University of the Basque Country, UPV00172.310-14456/2002, and Ministerio de Educación y Ciencia, FIS2004-01626.

\section{APPENDIX A: DETAILED COMPUTATION OF} EQ. (45)

Using the definition (33), we have that $\forall \varphi \in \mathcal{D}^{\prime}\left(\mathbb{R}^{4}\right)$

$$
\begin{aligned}
\left(\partial_{\mu} \hat{\theta}_{R}^{\mu \nu}, \varphi\right)= & -\left(\theta_{R}^{\mu \nu}, \partial_{\mu} \varphi\right) \\
= & \lim _{\epsilon \rightarrow 0}\left\{-\int_{\rho \geq \epsilon} d^{4} x \Theta_{R}^{\mu \nu}(x) \partial_{\mu} \varphi(x)\right. \\
& \left.+\frac{e^{2}}{2 \epsilon} \int_{-\infty}^{\infty} d \tau\left[v^{\mu} v^{\nu}+\frac{1}{3} \hat{\eta}^{\mu \nu}\right] \partial_{\mu} \varphi\right\} .
\end{aligned}
$$

Since $\Theta_{R}^{\mu \nu}(x)$ is summable for $\rho \geq \epsilon$, the first integral on the r.h.s. becomes

$$
\begin{aligned}
I_{1} \equiv & \int_{\rho \geq \epsilon} d^{4} x \partial_{\mu} \Theta_{R}^{\mu \nu}(x) \varphi(x) \\
& -\int_{\rho \geq \epsilon} d^{4} x \partial_{\mu}\left[\Theta_{R}^{\mu \nu}(x) \varphi(x)\right] .
\end{aligned}
$$

The first term vanishes because there is no current in $\rho \geq \epsilon$ and, applying Gauss theorem, the second one yields

$$
\epsilon^{2} \int_{-\infty}^{\infty} d \tau \int d^{2} \Omega \Theta_{R}^{\mu \nu}(\rho=\epsilon)\left[n_{\mu}+\epsilon(a n) k_{\mu}\right] \varphi\left(z^{\lambda}+\epsilon k^{\lambda}\right),
$$

where $(a n) \equiv a^{\lambda} n_{\lambda}$ and $d^{2} \Omega$ is the solid angle element. Using then Eq. (29) and the Taylor expansion [26] $\varphi(z+$ $\epsilon k)=\varphi(z)+\epsilon k^{\lambda} \partial_{\lambda} \varphi(z)+\frac{1}{2} \epsilon^{2} k^{\mu} k^{\lambda} \partial_{\mu \lambda} \varphi(z)+\mathrm{O}\left(\epsilon^{3}\right)$, Eq. (A2) yields

$$
\begin{aligned}
I_{1}= & \int_{-\infty}^{\infty} d \tau \int \frac{d^{2} \Omega}{4 \pi}\left\{-\frac{e^{2}}{2 \epsilon^{2}}\left(v^{\nu} \epsilon(a n)\left[\varphi+\epsilon k^{\lambda} \partial_{\lambda} \varphi\right]\right.\right. \\
& \left.+n^{\nu}[1+\epsilon(a n)]\left[\varphi+\epsilon k^{\lambda} \partial_{\lambda} \varphi+\frac{1}{2} \epsilon^{2} k^{\mu} k^{\lambda} \partial_{\mu \lambda} \varphi\right]\right) \\
& +\frac{e^{2}}{\epsilon}\left[a^{\nu}-\left(a^{2}\right) n^{\nu}\right]\left[\varphi+\epsilon k^{\lambda} \partial_{\lambda} \varphi\right] \\
& \left.+e^{2}\left[a^{2}-(a n)^{2}\right] k^{\nu} \varphi\right\}+\mathrm{O}(\epsilon) .
\end{aligned}
$$

On integration with respect to $d^{2} \Omega$ and using that

$$
\begin{gathered}
\int d^{2} \Omega n^{\nu}=\int d^{2} \Omega n^{\nu} n^{\mu} n^{\lambda}=0 \text { and } \\
\int d^{2} \Omega n^{\nu} n^{\mu}=\frac{4 \pi}{3} \hat{\eta}^{\nu \mu},
\end{gathered}
$$

we arrive at

$$
\begin{aligned}
I_{1}= & \frac{e^{2}}{2 \epsilon} \int_{-\infty}^{\infty} d \tau\left(a^{\nu} \varphi-\frac{1}{3} \hat{\eta}^{\mu \nu} \partial_{\mu} \varphi\right) \\
& +\frac{2 e^{2}}{3} \int_{-\infty}^{\infty} d \tau\left[a^{2} v^{\nu}-\dot{a}^{\nu}\right] \varphi .
\end{aligned}
$$

It is straightforward to check that the first term on the r.h.s. exactly compensates the second term on the r.h.s. in (A1). Therefore we have

$$
\partial_{\mu} \hat{\theta}_{R}^{\mu \nu}=\frac{2}{3} e^{2} \int d \tau\left[a^{2} v^{\nu}-\dot{a}^{\nu}\right] \delta(x-z(\tau)) .
$$

\section{APPENDIX B: THE CONSTITUTIVE RELATION}

We have to solve Eq. (76)

$$
\begin{aligned}
& (l \alpha+p \rho+r x) \mu_{\alpha}+(x-\alpha) \mu_{\rho}+\alpha(1+\mu) \mu_{x} \\
& \quad=\alpha(x-\alpha)
\end{aligned}
$$

with the "initial condition" $\mu(0,0,0)=0$ and $l, p, r$ constants.

It is easily seen that this equation admits a perturbative solution like

$$
\mu=\sum_{n=1}^{\infty} \mu^{(n)}
$$

$\mu^{(n)}$ being a polynomial in the variables $\alpha, \rho, x$ which is homogeneous and has degree $2 n$. If we write

$$
\hat{D} \equiv(l \alpha+p \rho+r x) \partial_{\alpha}+(x-\alpha) \partial_{\rho}+\alpha \partial_{x}
$$

then Eq. (B1) yields the hierarchy:

$$
\hat{D} \mu^{(1)}=\alpha(x-\alpha)
$$

$$
(n>1) \quad \hat{D} \mu^{(n)}=-\sum_{s=1}^{\infty} \mu^{(n-s)} \alpha \partial_{x} \mu^{(s)} .
$$

The lowest order is relatively easy to solve and yields:

$$
\begin{aligned}
\mu= & -\frac{1}{2 \Delta}\left[(p-r) \alpha^{2}+p^{2} \rho^{2}+\left(r^{2}+p+r l\right) x^{2}\right. \\
& -2 p \alpha x+2 r p \rho x]+\mathrm{O}(4)
\end{aligned}
$$

with $\Delta=l(p-r)-p$. 
[1] P. A. M. Dirac, Proc. R. Soc. A 167, 148 (1938).

[2] F. Rohrlich, Classical Charged Particles (AddisonWesley, Reading, MA, 1965).

[3] E. G. P. Rowe, Phys. Rev. D 12, 1576 (1975)

[4] D. V. C. Teitelboim and V. Weert, Riv. Nuovo Cimento 3, 1 (1980).

[5] M. Ibison and H. E. Puthoff, J. Phys. A 34, 3421 (2001).

[6] R. Blanco, Phys. Rev. E 51, 680 (1995).

[7] F. Rohrlich, Am. J. Phys. 65, 1051 (1997).

[8] E. J. Moniz and D.H. Sharp, Phys. Rev. D 10, 1133 (1974).

[9] E. J. Moniz and D.H. Sharp, Phys. Rev. D 15, 2850 (1977).

[10] E. J. Moniz and D. H. Sharp, Am. J. Phys. 45, 75 (1977).

[11] A. Valentini, Phys. Rev. Lett. 61, 1903 (1988).

[12] A.D. Yaghjian, Relativistic Dynamics of a Charged Sphere (Springer-Verlag, Berlin, 1992).

[13] E. Kerner, J. Math. Phys. (N.Y.) 6, 1218 (1965).

[14] J. L. Sanz, J. Math. Phys. (N.Y.) 20, 2334 (1979).

[15] L. Bel, in Relativistic Action at a Distance: Classical and Quantum Aspects, edited by J. Llosa (Springer-Verlag, Berlin, 1982), p. 21.

[16] J. M. Aguirregabiria, J. Phys. A 30, 2391 (1997).

[17] J. M. Aguirregabiria, A. Hernández, and M. Rivas, J. Phys. A 30, L651 (1997).

[18] J. M. Aguirregabiria, Ll. Bel, A. Hernández, and M. Rivas, Comput. Phys. Commun. 116, 95 (1999).

[19] H. Spohn, Europhys. Lett. 50, 287 (2000).
[20] P. Pearle, in Electromagnetism Paths to Research, edited by D. Teplitz (Plenum, New York, 1982), Chap. 7.

[21] D. Villarroel, Phys. Rev. A 55, 3333 (1997).

[22] E. G. P. Rowe, Phys. Rev. D 18, 3639 (1978).

[23] A. Lozada, J. Math. Phys. (N.Y.) 30, 1713 (1989).

[24] E. G. P. Rowe, Nuovo Cimento B 73, 226 (1983).

[25] E. Honig and G. Szamosi, Phys. Lett. A 93, 319 (1983).

[26] T. M. Apostol, Mathematical Analysis (Addison-Wesley, Reading, MA, 1981), 2nd ed.

[27] J. L. Synge, Relativity: The Special Theory (NorthHolland, Amsterdam, 1965).

[28] I. M. Gel'fand and G. E. Shilov, Generalized Functions (Academic, New York, 1964).

[29] V.S. Vladimirov, Equations of Mathematical Physics (Mir, Moscow, 1984).

[30] M. Mathison, Proc. Cambridge Philos. Soc. 36, 331 (1940).

[31] P. Havas, in Proceedings of International School of Physics "Enrico Fermi", Course LXVII, edited by J. Ehlers (North-Holland, Amsterdam, 1977).

[32] W. G. Dixon Special Relativity. The Foundation of Macroscopic Physics (Cambridge University Press, Cambridge, England, 1978).

[33] E. C. G. Sudarshan and N. Mukunda, Classical Dynamics: A Modern Perspective (Wiley, New York, 1974).

[34] A. Ori and E. Rosenthal, Phys. Rev. D 68, 041701(R) (2003). 